\title{
Experimenting with Mathematical Biology
}

\author{
Rebecca Sanft and Anne Walter
}

\begin{abstract}
St. Olaf College recently added a Mathematical Biology concentration to its curriculum. The core course, Mathematics of Biology, was redesigned to include a wet laboratory. The labs required students to collect data and implement the essential modeling techniques of formulation, implementation, validation, and analysis. The four labs investigated population growth, enzyme kinetics, glucose-insulin feedback and random walks/diffusion. Based on assessment data, having the lab and class juxtaposed was an effective way to reinforce mathematical concepts and encourage collaboration among students with different majors. We discuss key factors that permitted this course's development, the continuing challenges and how this model might be adapted to other venues.
\end{abstract}

Keywords: integrated curricula, biology, wet laboratory, applied mathematics 